\title{
Soil-Structure Interaction under the Effect of High Groundwater Table
}

\author{
Ilhan Burak Duran \\ Department of Civil Engineering, Istanbul Kultur University, Istanbul, Turkey \\ Email: b.duran@iku.edu.tr
}

How to cite this paper: Duran, I.B. (2017) Soil-Structure Interaction under the Effect of High Groundwater Table. Open Journal of Civil Engineering, 7, 561-569. https://doi.org/10.4236/ojce.2017.74038

Received: November 22, 2017 Accepted: December 26, 2017 Published: December 29, 2017

Copyright $\odot 2017$ by author and Scientific Research Publishing Inc. This work is licensed under the Creative Commons Attribution International License (CC BY 4.0).

http://creativecommons.org/licenses/by/4.0/

\begin{abstract}
This paper gives an account of a study performed for the raft foundation of a commercial building of considerable height and area. A raft $175 \mathrm{~m}$ long was designed without due consideration to the buoyancy effect due to high groundwater table as the building is near the sea. Although the raft was designed as an uninterrupted system, the designer used different, and insufficient, thicknesses for the foundation in order to lower costs. A 3D study was subsequently undertaken to analyze the settlements of the raft using finite elements. There was reasonable agreement between the computed and the measured settlements. However, the front block of the raft was observed to float as soon as pumping for lowering of the groundwater table was halted. This instigated the analyzers to tie this portion of the raft to the surrounding piled curtain that was used for excavation of the foundation pit, by means of reinforced concrete beams. The computations show that the heave of the floor was restrained at acceptable levels. It is planned to stop pumping in the near future and compare the computed and measured vertical movements.
\end{abstract}

\section{Keywords}

Finite Element, Raft, 3D Analysis, Buoyancy, Settlement

\section{Introduction}

It is nowadays standard practice to found high rise buildings on piled rafts mostly by unjustified approaches to the issue. This over conservative approach can partly be attributed to excessive reaction to the effects of potential earthquake damage as well as the general tendency to employ elastic methods for the calculation of footings. These methods emanate from the Winkler approach where only two parameters $\sigma$ all and ks are needed (Winkler, 1867) [1].

The Winkler hypothesis is the source of several defects. The semi-infinite 
medium, on which the foundation is placed, is represented by independent and rarely interconnected springs. Soil layering and the effect of the Ground Water Table are ignored. The software using the Winkler hypothesis results in conservative design simply because the concept of "allowable stress" for the soil is not a realistic parameter.

The geotechnical engineer has, in the past 30 years, been provided by numerical solutions such as the method of finite elements which enable him to model the soil as a non-linear-heterogeneous medium as well as including the mechanism of soil-structure interaction in the analyses.

This paper gives an account of the effort to refine the design of a raft foundation for a hotel building comprising three blocks that had been designed with an excessive amount of piles. In addition, a description of a solution foreseen to counteract the floating effect of rising Ground Water Table on the raft that has been cleared of the redundant piling system is given. A comparison of the computed and measured settlements is also made.

\section{Design of the Raft}

Floating effects that are the result of rising GWT can reach significant levels which may inflict damage on the structural members. A secondary effect of this movement is the problem of destroyed water insulation. Increasing numbers of rafts are nowadays being designed monolithically to overcome this problem, despite the fact that the structural engineers are yet reluctant to adapt to this kind of solution. Another proposal is to place the building blocks on this raft which have expansion joints among them. This however has been found to be ineffective in several cases.

The raft for a hotel building with three blocks A, B and C that will sit on a 175 $\mathrm{m}$ long raft was designed to act monolithically (Figure 1). The front block consists of two basements and no superstructure. Accordingly, the raft here had been designed thinner than the rest of the blocks. Block-B in the middle is the heaviest part with 2 basements, ground floor and 17 stories and was provided with the thickest part of the raft. Block-A has a low rise part as well as a 15 story tower next to it.

The writer of this paper was introduced to the project at this stage where a piled raft had already been proposed. A three-dimensional analysis of the raft was implemented in two stages. In the first part the amount of settlements of the raft and subsequent lifting due to the rising GWT was estimated. In the second part of the study the raft was connected to the piled curtain around the perimeter of Block-B by means of dowels and RFC beams. However, it was not easy to persuade the author of the project that piling was not essential for a successful raft performance.

The foundation soil consists of over consolidated clays overlying weak limestones with occasional beach sands on the top (Figure 2). The GWT is at a depth of $2 \mathrm{~m}$, the average level of the sea. 


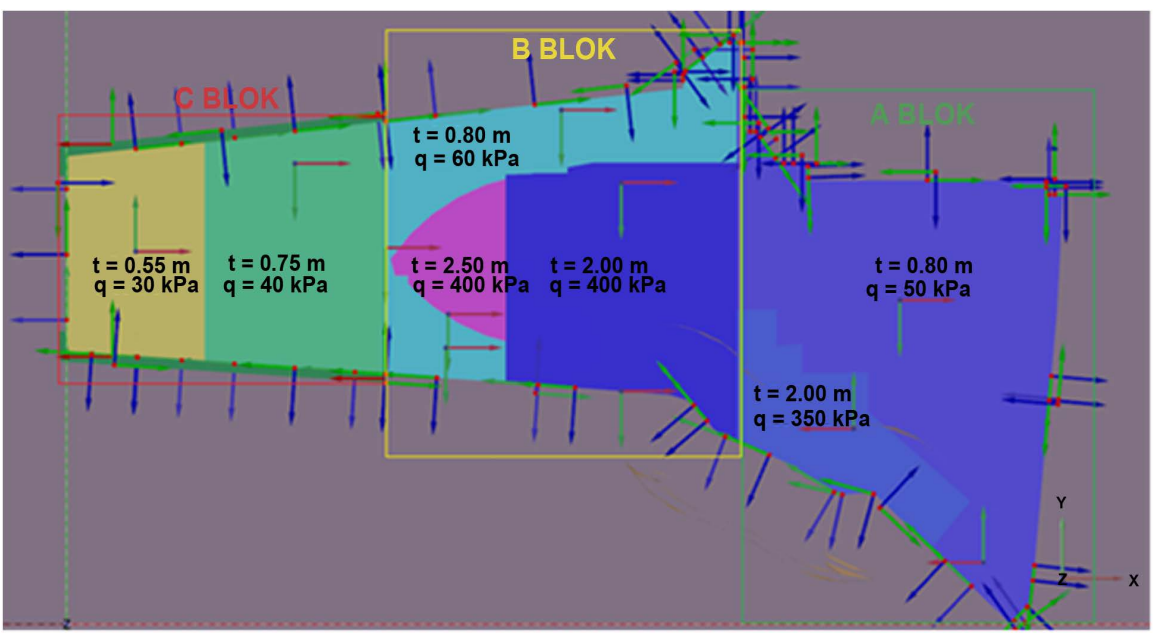

Figure 1. Geometry of the raft and load distribution.

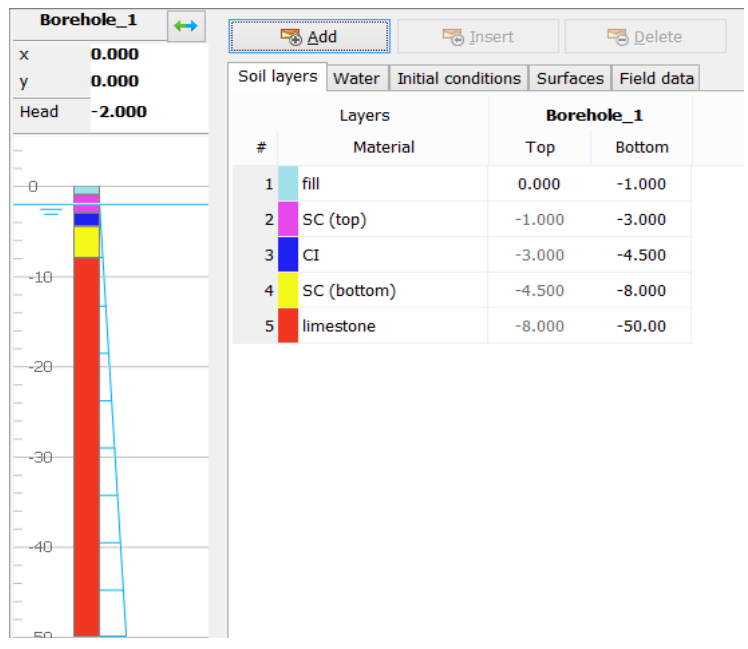

Figure 2. Soil profile used for geotechnical modelling.

Figure 4 shows that the settlements which were recorded regularly were well below critical levels despite the size of the raft.

The GWT was kept below the foundation level by means of pumping during the excavation of the pit. Although the operation was maintained following the completion of the basements, the owners expressed the wish that this should be discontinued in the long run. Accordingly, pumping was stopped in May 2013 to discover that B-Block was lifted by buoyancy and rose by about $170 \mathrm{~mm}$ (Figure 4). This caused slight damage along the expansion joints and pumping was resumed.

This process was modelled in three dimensions to see whether any damage would be inflicted on the structure.

\section{Modelling and Analysis}

It was necessary to employ the Autocad 3D to adapt the geometry of the structure to the FEM software. The surface geometry obtained was then transferred to 
the Plaxis 3D software which provided plate elements of varying thickness. In addition, the piled curtain with $0.8 \mathrm{~m}$ diameter contiguous piles supported by 3 rows of anchorages at a distance of $1.5 \mathrm{~m}$ from the basement walls were incorporated into the model (Plaxis 3D, 2013) [2].

It was found that the software did not perform initially, especially with the buoyancy effect of the GWT. It became necessary to improvise by lowering the GWT to below the basement and apply equivalent all-round pressure to the system. A further problem arose because the program joined all the forces applied from different directions into one. This problem was overcome by using different unit weights for the foundation elements representing the load from the superstructure and the weight of the foundation. This resulted in a lifting force being applied from the base only.

Two analyses were carried out. First, the movements of the raft were calculated under loads as well as the buoyancy effect. Second analysis was performed with the system under the influence of floatation forces being connected to the piled curtain around the perimeter by means of $1.5 \mathrm{~m}$ thick tie beams and saw the difference with the first analysis. The computation was done in the order,

- Modelling the initial conditions

- Activating the piled support system

- Excavation and dropping the GWT

- Zeroing the displacements and construction of vertical curtains around the basement

- Filling the space between the piles and the basement in 4 steps

- Application of the flotation effect

The problem encountered in the second phase of the calculations was the loss of convergence while attempting to fill the $1.5 \mathrm{~m}$ wide and $8.0 \mathrm{~m}$ deep gap between the piled curtain and the basement a one step. This had to be done in 4 steps which decreased the number of nodes by diminishing the number of elements required.

The second source of difficulty was found to be the necessity to adopt values of strength parameters for the fill material which are considerably higher than the actual values. This can be attributed to the fact that the analysis is aborted while calculating the settlement of Block-C: tensile stresses were established in this material due to the acting flotation effects. The increased rigidity of the fill secured uninterrupted running of the program. It was also noticed during the computations that the use of interface elements between the concrete plate elements and the soil volumetric elements were indispensable and should be clearly defined for successful modeling (Brinkgreve, et al., 2013) [3].

\section{Conclusion and Results}

Although it required considerable time and effort, reasonable results could be obtained in the three-dimensional analysis of a complicated raft-superstructure system under the influence of floatation effects with the following results. 


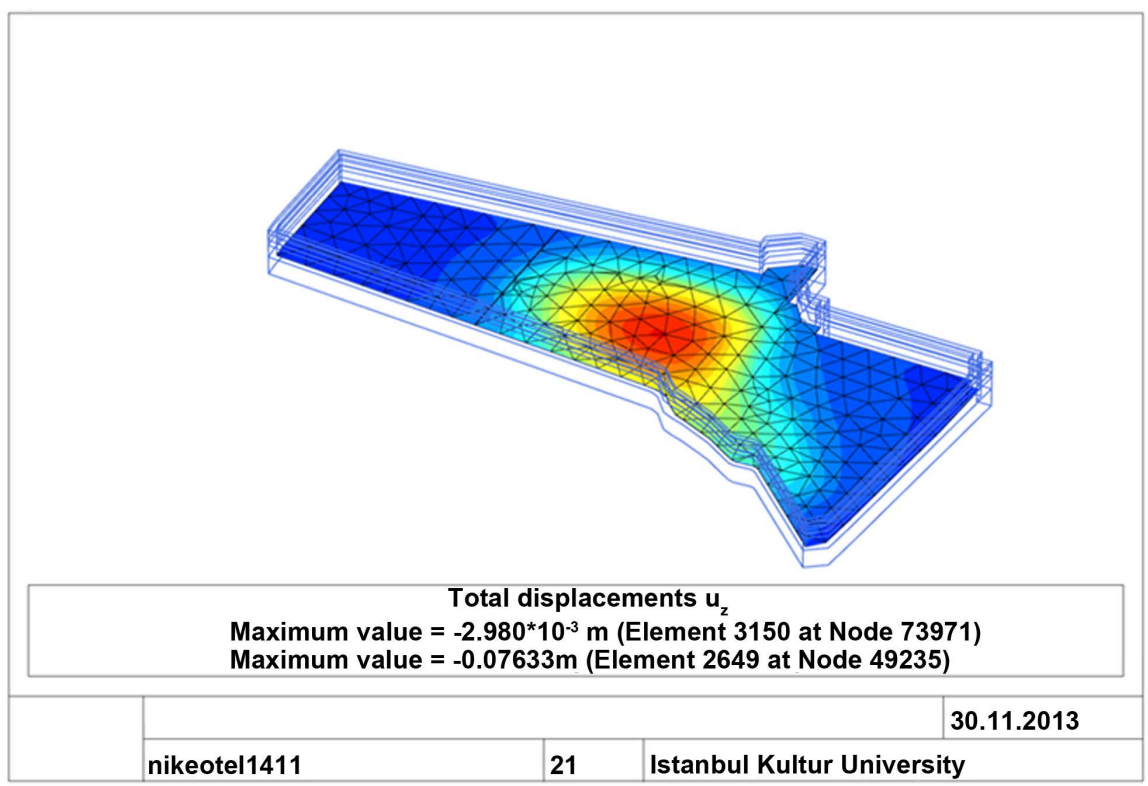

(a)

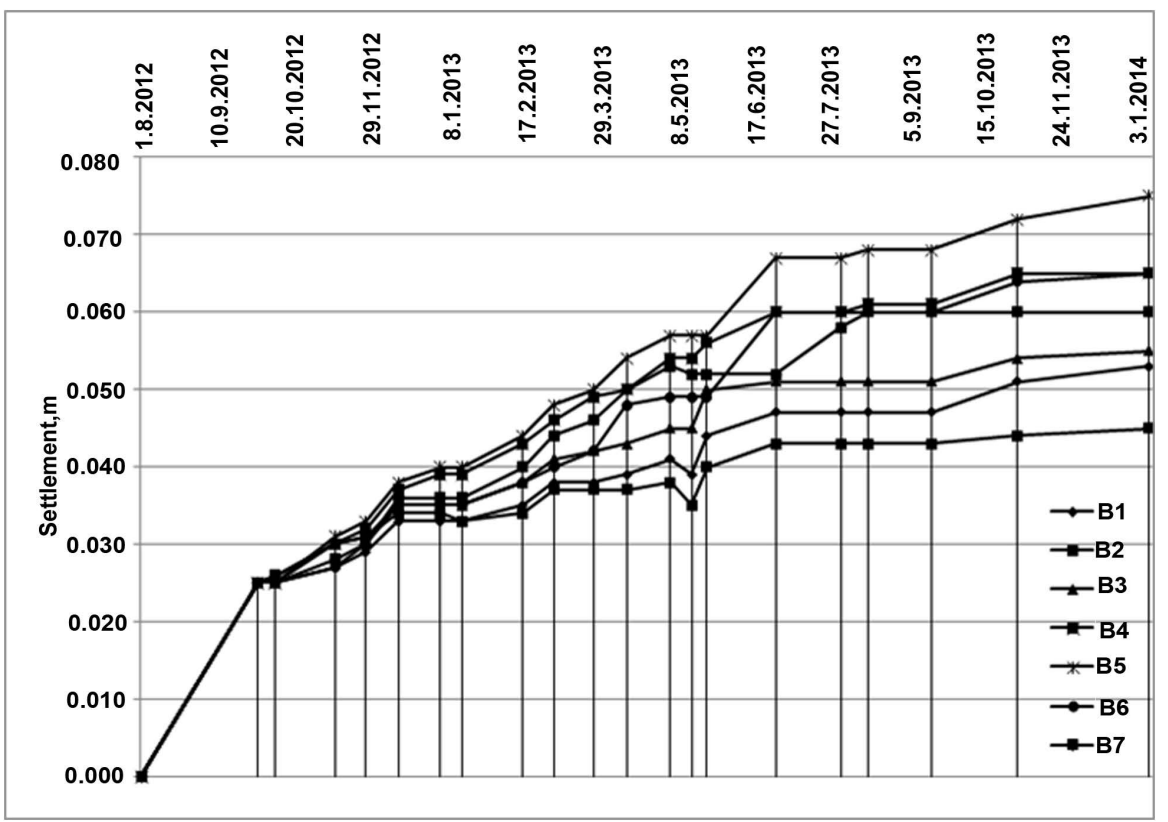

(b)

Figure 3. The results of FEM analysis and real-time settlement measurements b-block.

The system was analyzed as built in the first phase of computation. A heave of $170 \mathrm{~mm}$ was predicted following an excavation of $8.0 \mathrm{~m}$ for the raft. The maximum settlement of $76 \mathrm{~mm}$ under B Block was obtained as $76 \mathrm{~mm}$ prior to the application of the uplift force. This prediction was found to be consistent with the measured settlement (Figure 3).

A heave of $110 \mathrm{~mm}$ was measured after resetting all the deformations and applying a uniform pressure of $60 \mathrm{kPa}$, representing the hydrostatic force was applied under the C-Block (Figure 4). 


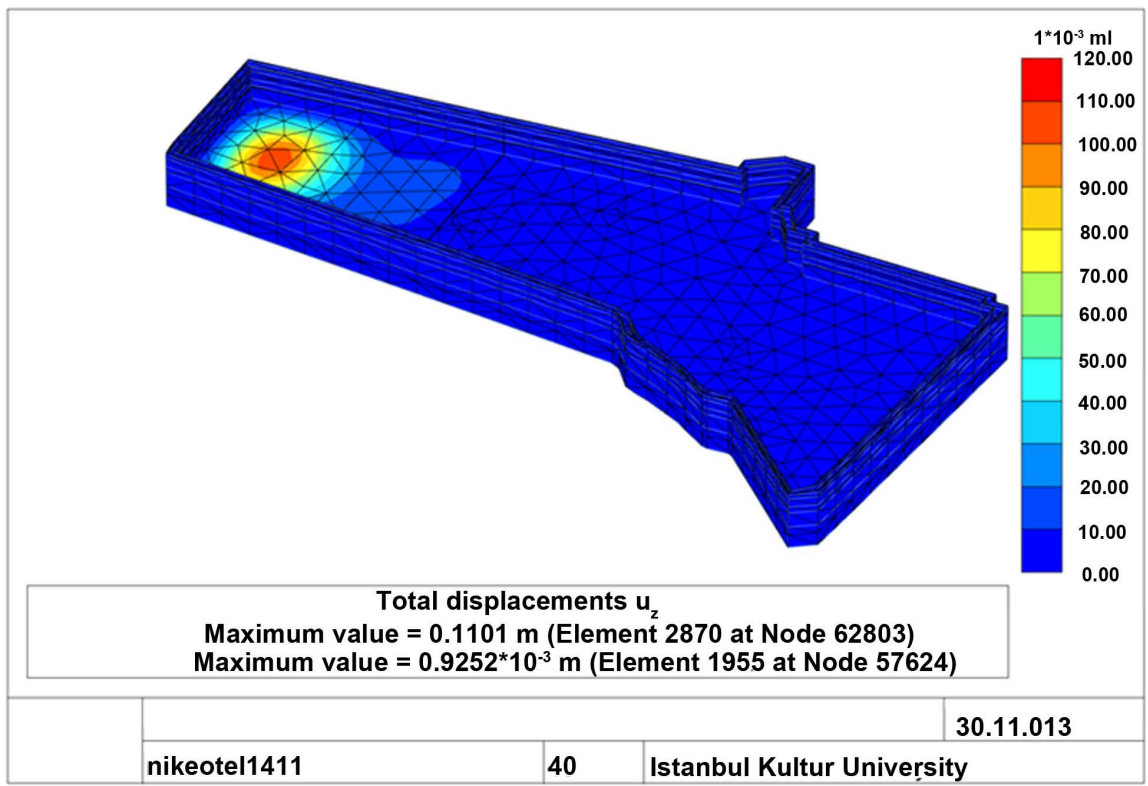

(a)

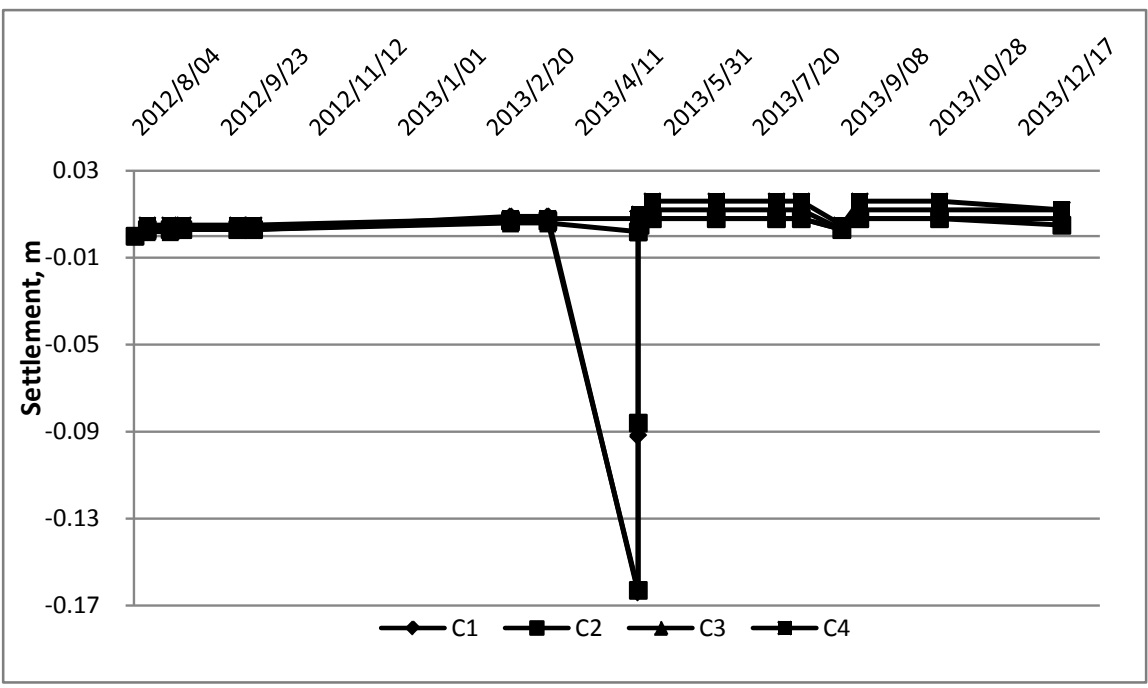

(b)

Figure 4. The Real-time computed and measured vertical movements of c-block under the influence of floatation effects (Spike indicates halting of pumping in 11 May 2013).

A further step in the computation was taken by inserting tie beams of $1.5 \mathrm{~m}$ length and height between the basement of C-Block and the piled curtain (Figure 5) to control and reduce the upwards movement. This reduced the vertical movements to an acceptable level of $8.0 \mathrm{~mm}$.

Pumping was halted after the application of the proposed stabilising measures and readings of the movements were recorded to see whether the recommended system performed satisfactorily.

Figure 6 and Figure 7 show that there is a close agreement between the computed values and the measured vertical movements. 


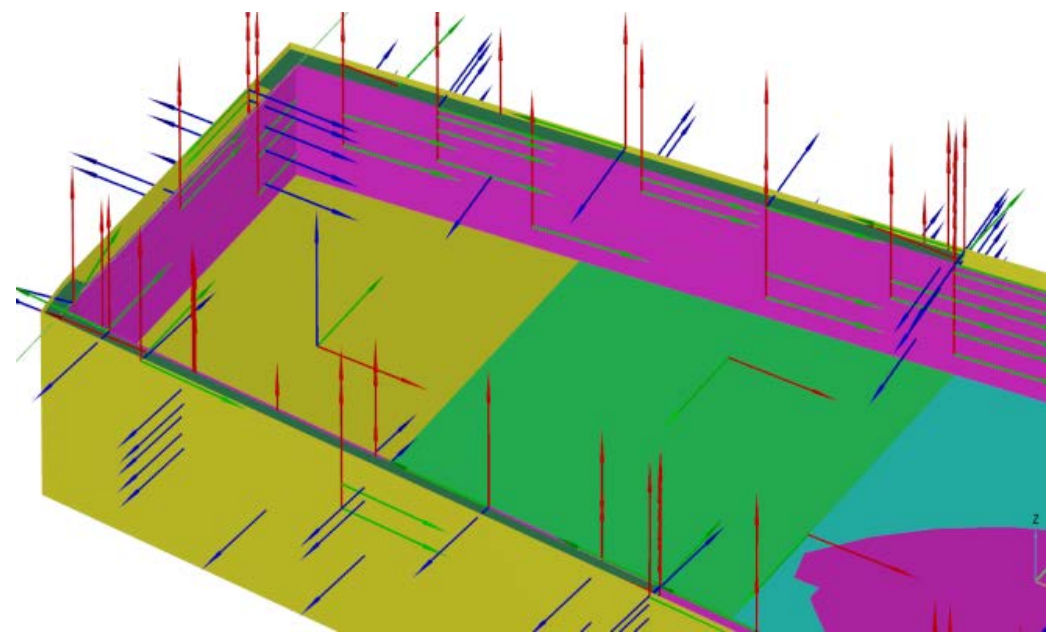

(a)

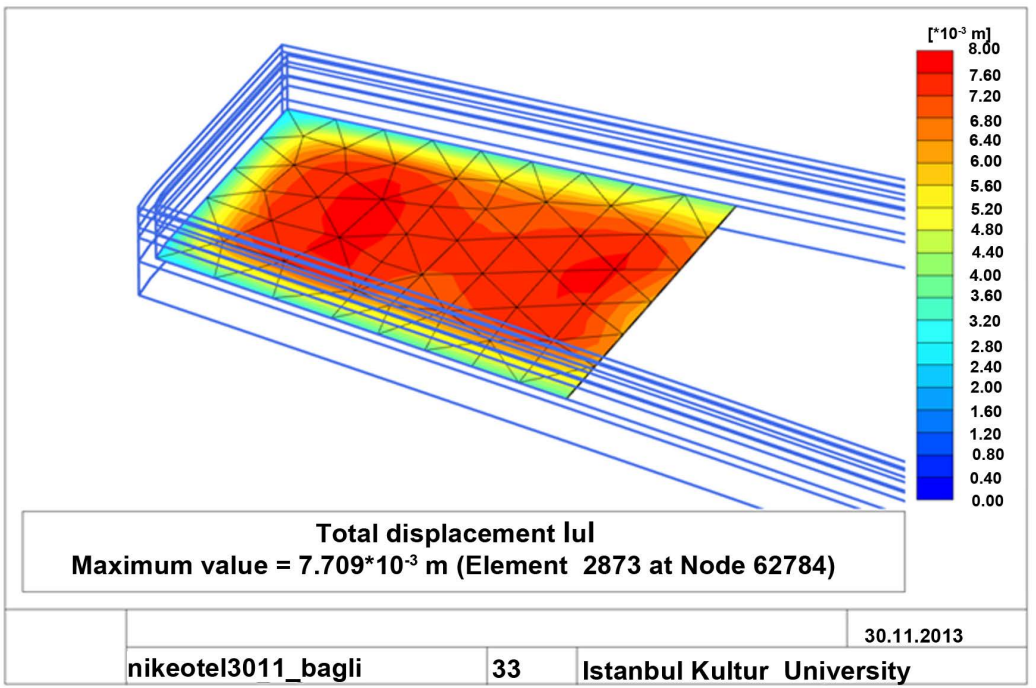

(b)

Figure 5. Activation of the tie beams between the basement and the piled curtain and its influence on the analysis.

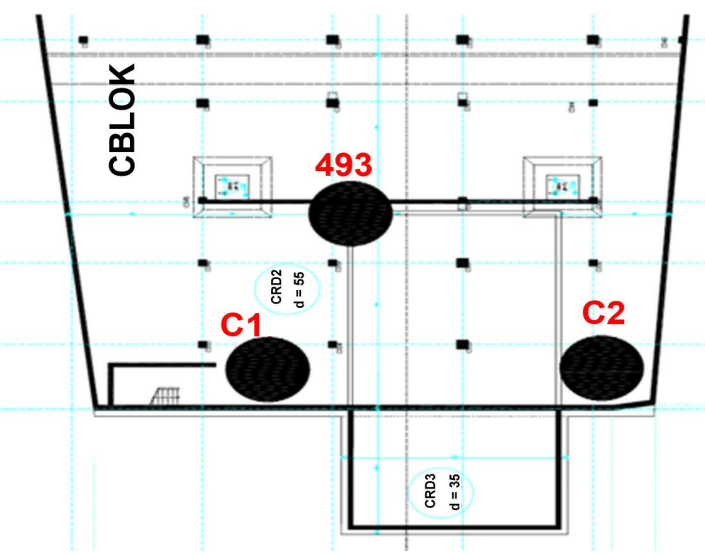

(a) 


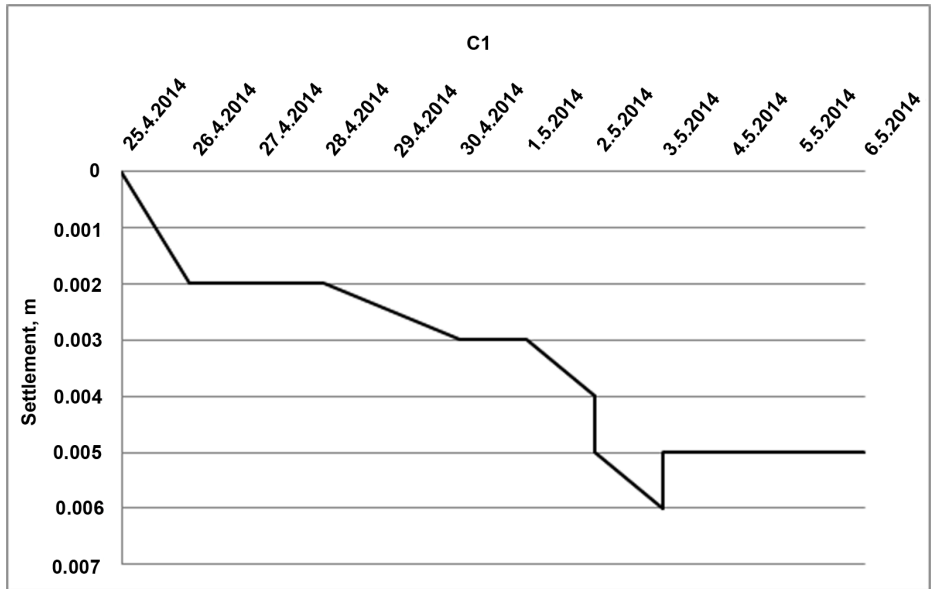

(b)

Figure 6. Settlement mesuring points under c-block and time-settlement curve for $\mathrm{C} 1$ under the influence of GWT.

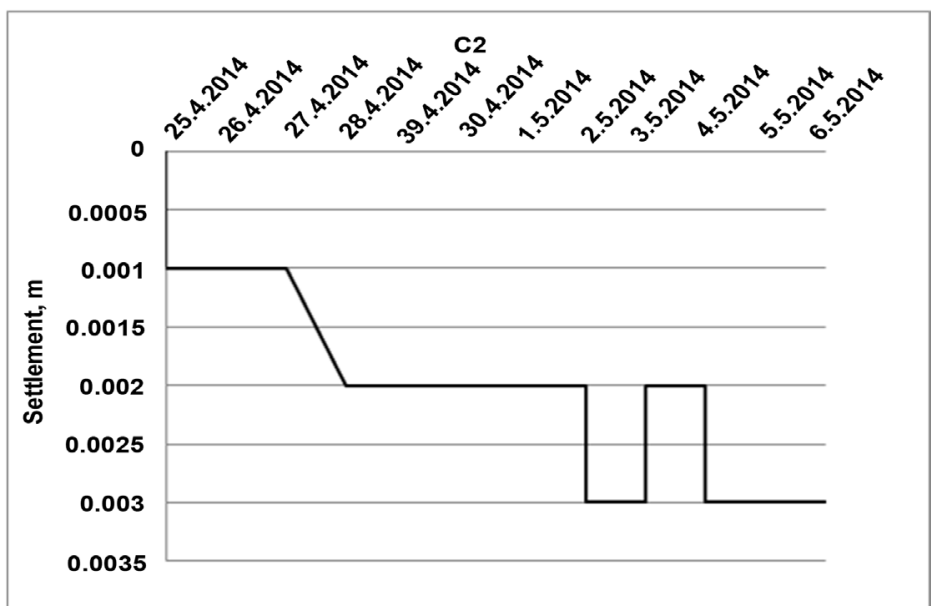

(a)

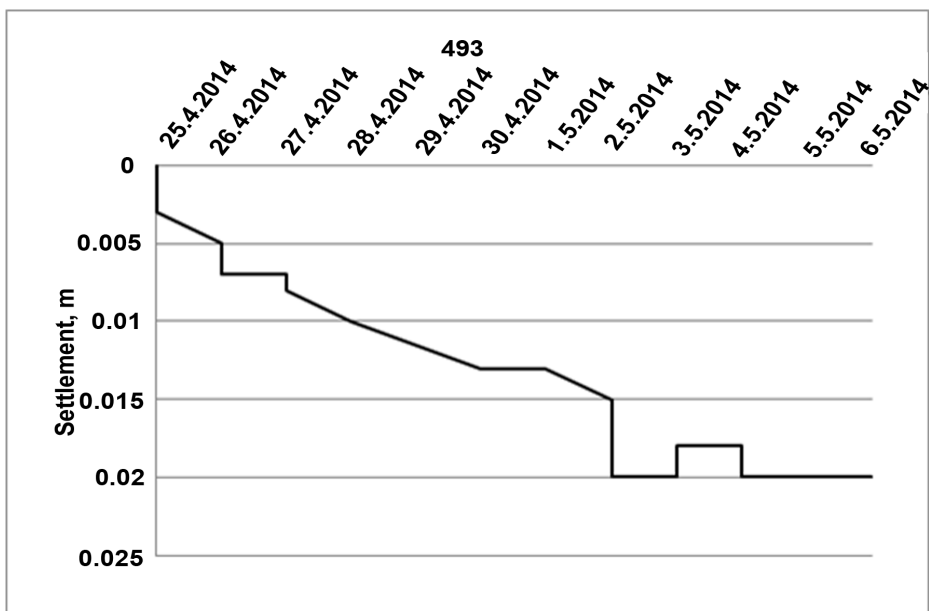

(b)

Figure 7. Time-Settlement Plot of Selected Points Under C-Block Under GWT Effect: C2 and 493. 
It can be concluded that a 3D study of raft-soil interaction is justified. Improvement can be achieved by advancing the study to soil-raft-superstructure interaction.

\section{References}

[1] Winkler, E. (1867) Die Lehre von Elastizitaet und Festigkeit, Praha.

[2] Plaxis 3D 2013 Material Models Manual.

[3] Brinkgreve, R.B.J., Engin, E. and Swolfs, W.M. (2013) Plaxis 3D, General Information Manual. 